\title{
Review of robotic manipulators and identification of the main problems
}

\author{
Ksenia I. Goryanina*, Aleksndr D. Lukyanov, and Oleg I. Katin \\ Don State Technical University, 344000 Rostov-on-Don, Russia
}

\begin{abstract}
One of the main elements of automation of industrial enterprises is the use of robotic systems consisting of mechanical manipulators and control systems. In recent years, the market of service robotics has been actively developing. The main part of the market of professional service robots in value terms is occupied by medical devices. Agriculture and logistics are also actively developing areas. The success of the automation systems implementation depends on the solution of complex scientific and technical problems, primarily in the following areas: machine vision; sensor networks; navigation systems. Thus, one of the fundamental problems, the solution of which largely depends on the success in creating the perfect adaptive and intelligent robots, is the use of such types of sensors of sensory information, which allow obtaining a sufficiently large amount of information about the problem environment in a short time. This is a problem of creating means of perception.
\end{abstract}

\section{Introduction}

In the modern world, the main direction of industry development is the automation of production. This contributes to the growth of its efficiency by improving the quality of produced products, as well as reducing the share of workers employed in various areas of production.

One of the main elements of automation of industrial enterprises is the use of robotic systems consisting of mechanical manipulators and control systems. The copying manipulators operated by the human operator are necessary when performing various works with radioactive materials. In addition, these devices are indispensable when performing work in space, under water, in chemically active environments [1 - 3]. Thus, industrial robots and copying manipulators are important components of modern industrial production. Moreover, today robotic manipulators have found their application not only in production, but also in everyday life as household helpers [4 - 7].

The main advantage of using robotic manipulators is the reduction of production costs. And the main drawback can be called high cost, but it is offset by an increase in the number of products. Another disadvantage is the need for services only from firms-manufacturers, which are often located abroad. But at the same time such manipulators are highly reliable [8-9].

\footnotetext{
* Corresponding author: gorianina.k@yandex.ru
} 
In recent years, the market of service robotics has been actively developing. The main part of the market of professional service robots in value terms is occupied by medical devices. Agriculture and logistics are also actively developing areas [10 - 12].

The rapid development of events in this area attests to the relevance of the topic under consideration. First, it is necessary to consider the state of the problem as a whole, so the purpose of the work is to review the basic directions of development of robotic manipulators.

\section{Overview of manipulators}

In the modern world, a robot manipulator is a mechanism like a human hand-a standard anthropomorphic robot manipulator. Any of the manipulators of an industrial robot is a universal device, usually having several axes of mobility and a flange for tool installation. The most common are remotely controlled "mechanical arms", which are fixed on a fixed or movable base.

Characteristics of manipulators differ, first of all, depending on the purpose of robots. For example, the hand-manipulator JACO Robot Manipulator [13] from Canadian developers Kinova has a strong grip of six connections with unlimited rotation around the axis. The arm of the manipulator is controlled by special software via PC, which is equipped with a USB input. The software interface gives the user full control, allows diagnosis. The weight of the robot is only $5.7 \mathrm{~kg}$, and the maximum load capacity is $1.5 \mathrm{~kg}$ and with a fully retracted hand $-1 \mathrm{~kg}$. Reach $90 \mathrm{~cm}$.

Hand manipulator American Advanced Robotic Manipulator (ARM 2.0) [14] the company RobotShop has six poles and an open control interface, reach, reaching in diameter 2.2 meters, which allows ensuring the realization of any projects. Each connection has its own position due to the assigned RC PWM signal. The manipulator has a high level of position control - all connections with a closed circuit operate in the position control mode. Its weight is $9.2 \mathrm{~kg}$ and its capacity is $4.5 \mathrm{~kg}$ with full expansion.

The robot of the new generation BAXTER RESEARCH ROBOT [15] from American engineers of Rethink Robotics Company is able to learn new actions, as well as to recognize objects even in a reversed state. It has seven degrees of freedom manipulators and performs works related to sorting, loading/unloading, packaging and unpacking of products.

Moving the joints of the robots is produced by actuators using absolute dates-pins or with the aid of servomotors. The price of such robots is quite high.

There are also analogues of Chinese manufacturers. For example, the robot manipulator 7 DOF [16] with remote control. It has seven degrees of freedom, moves on the chassis and has a good load capacity (up to $7 \mathrm{~kg}$ ) with its small weight of $1.5 \mathrm{~kg}$.

Robot manipulator $A 40$ [16] SZDoit has become very popular in the world and is characterized by high accuracy of work, production, speed and reliability. It does not tremble at work, performs movements smoothly thanks to the stepper motor with planetary drive.

The market is a two-handed robot arms is not so widespread, but increasing demand in recent talks about his future development. A bright representative is a two-handed warehouse robot Epson ProSix C4-A901S [17] Hitachi companies. The robot is equipped with two manipulators mounted on a scissor lift, which is mounted on a mobile base. Each manipulator is able to lift a weight of about $1 \mathrm{~kg}$. At the moment, this robot is very expensive because of what has not yet hit the open market.

Another representative is the two-handed robot chef Moley [18] Moley Robotics companies. The robot is able to handle almost all kitchen utensils and appliances, skilfully copying human movements. When cooking, the robot repeats the movements of the cook, 
previously recorded on the camera and loaded into his memory. The cost of robotic kitchen does not exceed the cost of kitchen furniture.

Described above and other robots-manipulators have their advantages and disadvantages. But at the same time an important problem is the organization of manipulator control with additional requirements to the characteristics of interaction (forces and moments) with the environment in the conditions of joint work of several types of sensors (tactile, force-moment, visual, etc.).

\section{Technical implementation tasks}

The problem of creating intelligent robotic means, partially or completely replacing a person in solving various problems, is connected with the need for practical implementation of technical systems, which, like a person, should be able to perceive information from the external environment with the help of appropriate sensors, process and" comprehend " this information, develop solutions and sequences of necessary actions, as well as have the ability to operate, uncertain or even contradictory information about the external situation and at the same time complete the task. For the practical creation of such systems, it is necessary to solve a range of extremely complex technical problems and to develop a wide range of technical means and devices.

The success of the automation systems implementation depends on the solution of complex scientific and technical problems, primarily in the following areas:

- machine vision is an essential element of the robot's artificial intelligence;

- network of sensors make the robot's behaviour more adaptive;

- navigation systems: improving positioning accuracy and the ability to navigate within enclosed spaces.

Thus, one of the fundamental problems, the solution of which largely depends on the success in the creation of perfect adaptive and intelligent robots, is the use of such types of sensor information sensors that allow you to obtain a sufficiently large amount of information about the problem environment in a short time. This is a problem of creating means of perception.

\section{Prospects for solving the problem}

\subsection{Current state of Affairs with sensor systems of robotic anthropomorphic manipulators}

Considering the types of sensitivity that the human hand has, the following basic feelings can be distinguished: touch, feelings of temperature, weight, effort, movement and orientation. And some of these feelings have "graduation of feelings", for which individual sensory systems are responsible.

In the existing manipulators, as a rule, only feelings of orientation (or rather, the relative position of the manipulator elements relative to each other) and, sometimes - a sense of effort/weight due to the control of the moment of forces (usually - the magnitude of the current of the manipulator drives) are realized.

All this naturally (and significantly) limits the sensitive capabilities of existing robotic manipulators and makes their use in everyday life and special areas limited. 


\subsubsection{Promising areas for enhanced sensitivity}

If we consider the feelings that should / preferably have a "universal" robotic manipulator, you should pay attention to the following list, something copying, and in some ways complementary to the list of the previous section:

1. Touch: sense of touch; vibration sense; sense of pressure; feeling wet/dry; proximity sense (touch sensor).

2. Temperature sense: contact temperature sense; remote sense of temperature.

3. Sense of weight: static weight sense; dynamic sense of weight.

4. Sense of effort.

5. Sense of movement.

6. Sense of orientation: sense of relative orientation; sense of absolute orientation; sense of the absolute turn/change position.

7. The sense of the magnetic field: feeling of a constant magnetic field; sense of an alternating magnetic field.

8. Sense of colour.

9. Sense of consistency (dense/monolithic, porous, mesh, etc.).

10. Sense of distance.

If focus on the proposed development of dual-use manipulator of household / special purpose, then, as it decreases, its sensor systems should provide the possibility of the following concerted actions:

1. Move the manipulators without colliding with the surrounding objects.

2. Consistent movement/placement of manipulators with the exception of the possibility of collision (finding the elements of the manipulator within the same volumes)

3. Consistent movement / placement of manipulators to ensure their interaction. In this case are governed by the mutual displacement of the elements of the manipulator relative to each other.

4. Consistent movement / placement of manipulators relative to held and moved or surrounding objects.

5. Holding objects with one force, located in a given range, for the purpose of manipulation.

6. Consistent holding of objects by two manipulators, with the force in a given range, in order to carry out manipulations.

7. Determining the temperature of an object clamped in the manipulator or an object that the manipulator is approaching

8. Determine the colour of the object to which the manipulator is approaching.

\subsection{The list of sensors for their placement on the perspective robotic manipulator}

To meet the above requirements, it is proposed to add the following sensors to the existing sensor system of the robotic manipulator:

The sensors of the orientation:

- absolute three-axis acceleration sensor (MEMS-type);

- three-axis gyroscope (MEMS-type).

These sensors of the orientation system are installed on each finger of the manipulator (on its last phalanx, ideally - on each phalanx), and on each previous link, providing their absolute orientation in the gravitational field of the Earth and measuring the rotation of the manipulator elements relative to the fixed coordinate system associated with its base.

Temperature sensor:

- surface temperature sensor; 
- sensor-pyrometer for remote temperature measurement.

As a surface temperature sensor, it is advisable to use low-inertia film sensors. These sensors should be on the surface of the inner side of the last phalanx of the manipulator fingers. The sensor-pyrometer can be built into the tip of one finger on one or both manipulators, and can be located in another arbitrary place. For example-on the palm, or on the joint of one of the phalanges of the finger.

Magnetic field sensor:

sensitive element of the sensor (analogue Hall sensor);

- active element of the sensor (permanent magnet or micro-winding of the electromagnet).

The use of magnetic sensors consisting of a sensing element and a permanent or electromagnet can be promising for determining the mutual orientation of the manipulator fingers when they are working at a short distance. At the same time, the use of high-speed Hall sensors and electromagnets with different modulation of the magnetic field will allow to determine the mutual approximation with different parts of the manipulator in the case when visual control is difficult or not possible.

Distance sensors:

- IR laser proximity/distance sensor;

- ultrasonic distance sensor.

The use of distance sensors built into the fingers of the manipulator will allow to solve the problem of determining the exact distance at a short distance, for example, to capture an object. At the same time, the IR laser sensor is more promising both because of its integral design and miniature size, and because of its greater accuracy. However, it is more susceptible to surface contamination than ULTRASONIC sensors.

Proximity and touch sensor:

- capacitive touch sensor;

- resistive / thin-film contact strain gauge.

The touch sensor can be used to determine the close proximity to any object (distance from $1 \mathrm{~mm}$ and less when the IR laser sensor stops working). Sensors, because of their simplicity, can be placed in several places on the inner surface of the manipulator brush, and used for capturing and fixing various objects. These sensors can be supplemented with contact sensors located on the surface of the manipulator (in the form of a coating - skin), and changing their properties at low deformation. This can be, for example, sensors such as "film keyboard", fixing "force" contact with the subject. They can be placed in "arrays" with dynamic polling/scanning by analogy with the membrane keyboard.

Colour sensor:

- RGB-colour sensor based on the matrix of phototransistors.

This sensor may be of limited use for determining the colour of objects directly in front of the manipulator. Set - similar to a remote pyrometer. Compared to the video camera, it has smaller size, cost and required computing resources.

Humidity sensor:

- MEMS humidity sensor type SHT-21 or similar.

It is placed on the tip of one of the fingers (possibly - the little finger, as the "safe") and allows you to assess the humidity near the sensor.

\subsection{The list of sensors for their placement on the perspective robotic manipulator}

Most of the above sensors are available as MEMS integrated circuits with access to digital bus type $\mathrm{I} 2 \mathrm{C}$ and/or SPI, providing a simple connection with modern interfaces of 
computing devices. Some types of sensors (primarily - tactile) will require the development of their own control circuits, but it does not present significant difficulties.

The greatest difficulty is seen in the processing and coordination of information obtained from this heterogeneous array of sensors, its analysis, and the development of control actions. Its solution will require both serious theoretical studies on the design of the automatic control system and significant costs for the implementation of the developed algorithms in the form of effective program code.

\section{Conclusions}

One of the main elements of automation of industrial enterprises is the use of robotic systems consisting of mechanical manipulators and control systems. The advantage of using robotic manipulators is, first of all, the reduction of production costs. And the main drawback can be called high cost, but it is offset by an increase in the number of products.

In recent years, the market of service robotics has been actively developing. The main part of the market of professional service robots in value terms is occupied by medical devices. Domestic robots are becoming more and more popular. Also actively developing direction-mi are agriculture and logistics.

One of the fundamental problems, the solution of which largely depends on the success in the creation of perfect adaptive and intelligent robots, is the use of such types of sensor information sensors that allow you to obtain a sufficiently large amount of information about the problem environment in a short time.

\section{References}

1. Available at: https://robotbaza.ru

2. N. N. Elyash. Fundamentals of robotics: tutorial (lecture notes) (2016)

3. P. N. Belyanin. Industrial robotics (Machine Building, Russia, 1975)

4. Available at: https://shop.robotclass.ru

5. Available at: http://www.prorobot.ru/forum/

6. Available at: http://roboforum.ru

7. Available at: http://www.nrec.ri.cmu.edu/projects/cargo/

8. Available at: http://spectrum.ieee.org

9. Available at: http://www.popularmechanics.com/technology/engineering/ robots $/ 2169012$

10. Robotics 2020. Multi-Annual Roadmap. Available at: https:/eurobotics.net/cms/upload/press/2015/files/Multi-Annual_Roadmap2020_ICT24_Rev_B_full.pdf

11. A Roadmap for US Robotics. Available at: https://parasol.tamu.edu/volzfest/panelstalks/VolzFestschrift-Talk-Christensen.pdf

12. Available at: http://pressria.ru/pressclub/20140603/949286799.html

13. Available at: http://www.roboticmagazine.com/robot-review/jaco-robot-arm-2

14. Available at: http://www.robotshops.ru

15. Available at: https://www.rethinkrobotics.com/baxter/

16. Available at: https://motocarrello.ru/jelektrotehnologii/roboty/1769-robotmanipuljator.html\#h7--1-s6-6dof-6---abb----4--mg996r--2--mg90s 
17. Available at: http://www.robogeek.ru/promyshlennye-roboty/hitachi-razrabotaladvuhrukogo-skladskogo-robota

18. Available at: https://hi-news.ru/technology/dvurukij-robot-povar-moley-zamenitxozyajku-na-kuxne.html 